\title{
Description of Spherical Aberration and Coma of a Microlens Using Vector Diffraction Theory
}

\author{
Glen D. Gillen ${ }^{a}$ and Shekhar Guha ${ }^{b}$ \\ ${ }^{a}$ Air Force Research Laboratory, Materials and Manufacturing Directorate, Anteon \\ Corporation, Dayton, Ohio, 45431, USA; \\ ${ }^{b}$ Air Force Research Laboratory, Materials and Manufacturing Directorate, Wright-Patterson \\ AFB, Ohio, 45433, USA
}

\begin{abstract}
Light distributions of a plane wave refracted by a microlens are calculated using Kirchhoff vector diffraction theory. Numerical results for one and two-dimensional beam profiles and the onset and effects of spherical aberration and coma are investigated for different lens parameters.
\end{abstract}

Keywords: vector diffraction theory, microlenses, aberration

\section{INTRODUCTION}

Ray-tracing and geometrical optics are traditionally used to locate the focal regions of lenses. Although these techniques are useful for quick calculations of focal distances and the magnitudes and regions of spherical aberration and coma, they are limited if the vector components of the light fields and/or detailed beam distributions are desired. For example, in the field of optical trapping and manipulation of cold atoms, it is desirable to have precise knowledge of the field components of tightly focused laser beams. The trapping of cold atoms using the dipole radiation force was first observed in 1978, by Bjorkholm, et al.. ${ }^{1}$ Recent work by Dumke et al. ${ }^{2}$ used a microlens array to focus laser light for the trapping of cold, neutral ${ }^{85} \mathrm{Rb}$ atoms. In this work we apply vector diffraction theory to the special case of a microlens in order to calculate light distributions and beam profiles in the focal region. Using the radiation and vector diffraction theory of Stratton, ${ }^{3}$ the diffraction integrals are numerically evaluated using average desktop computers. The onset and effects of spherical aberration and coma on one and two-dimensional beam profiles is investigated for various microlens and light parameters.

\section{THEORY}

The vector electromagnetic fields within a given source-free volume of space can be determined from the vector analog of Green's second identity using Stratton and Chu's method. ${ }^{4}$ The fields at point $\vec{r}$ can be expressed in terms of the field values on a surface, $S$, surrounding the point $\vec{r}$ using the formulae of Rothwell and $\mathrm{Cloud}^{5}$ as

$$
\begin{gathered}
\vec{E}(\vec{r})=-\int_{S} d a^{\prime}\left[-i \omega \mu\left(\hat{n}^{\prime} \times \vec{H}\left(\vec{r}^{\prime}\right)\right) G+\left(\hat{n}^{\prime} \times \vec{E}\left(\vec{r}^{\prime}\right)\right) \times \vec{\nabla}^{\prime} G+\left(\hat{n}^{\prime} \cdot \vec{E}\left(\vec{r}^{\prime}\right)\right) \nabla^{\prime} G\right], \\
\vec{H}(\vec{r})=-\int_{S} d a^{\prime}\left[i \omega \epsilon\left(\hat{n}^{\prime} \times \vec{E}\left(\vec{r}^{\prime}\right)\right) G+\left(\hat{n}^{\prime} \times \vec{H}\left(\vec{r}^{\prime}\right)\right) \times \vec{\nabla}^{\prime} G+\left(\hat{n}^{\prime} \cdot \vec{H}\left(\vec{r}^{\prime}\right)\right) \nabla^{\prime} G\right],
\end{gathered}
$$

where $\hat{n}^{\prime}$ is the normal to the input surface pointing into the volume, $\vec{r}^{\prime}$ denotes a point on the surface $S$ and $d a^{\prime}$ is the surface area element pointing in the direction of $\hat{n}^{\prime}$. The integrations extend over the input surface $S$ and the values of $\vec{E}\left(\vec{r}^{\prime}\right)$ and $\vec{H}\left(\vec{r}^{\prime}\right)$ in the integrands are those evaluated 'just inside' the volume of space after the input surface. The Green's function, $G$, used in Eqs. 1 and 2 is given by

$$
G=\frac{e^{-i k_{1} \rho}}{4 \pi \rho},
$$

Further author information: (Send correspondence to G. D. Gillen)

G. D. Gillen: E-mail: ggillen@anteon.com, Telephone: 19372523132

\footnotetext{
International Optical Design Conference 2006, edited by G. Groot Gregory,

Joseph M. Howard, R. John Koshel, SPIE Vol. 6342, 63420B,

(c) 2006 SPIE-OSA $\cdot 0277-786$ X/06/\$15 $\cdot$ doi: $10.1117 / 12.692198$
} 
where $k_{1}=2 \pi n_{1} / \lambda, n_{1}$ being the refractive index of the medium between $\vec{r}$ and $\vec{r}^{\prime}$, and

$$
\rho^{2}=\left|\vec{r}(x, y, z)-\vec{r}^{\prime}\left(x^{\prime}, y^{\prime}, z^{\prime}\right)\right|^{2} .
$$

When the surface separating the two media is spherical in shape, it is convenient to use a spherical coordinate system to evaluate the field integrals. For an incident plane wave traveling parallel to the $z$-axis, the values of the electromagnetic field components $\vec{E}\left(\vec{r}^{\prime}\right)$ and $\vec{H}\left(\vec{r}^{\prime}\right)$ 'just outside' of the spherical surface can be obtained from the surface curvature, refraction effects, and the appropriate Fresnel transmission coefficients. If $E_{o}$ denotes the amplitude of the electric field of the incident plane wave, assumed to be polarized along the $x$-direction,

$$
\vec{E}\left(\vec{r}^{\prime}\right)=E_{o} e^{-i p_{2} \cos \theta^{\prime}}\left(T_{p} \sin \theta_{t}^{\prime} \cos \phi^{\prime} \hat{r}+T_{p} \cos \theta_{t}^{\prime} \cos \phi^{\prime} \hat{\theta}-T_{s} \sin \phi^{\prime} \hat{\phi}\right),
$$

and

$$
\vec{H}\left(\vec{r}^{\prime}\right)=\frac{n_{1}}{Z_{o}} E_{o} e^{-i p_{2} \cos \theta^{\prime}}\left(T_{s} \sin \theta_{t}^{\prime} \sin \phi^{\prime} \hat{r}+T_{s} \cos \theta_{t}^{\prime} \sin \phi^{\prime} \hat{\theta}+T_{p} \cos \phi^{\prime} \hat{\phi}\right),
$$

where $Z_{o}=\sqrt{\frac{\mu_{o}}{\epsilon_{o}}}, p_{2}=2 \pi n_{2} R / \lambda$, and $R$ is the radius of curvature. $\theta^{\prime}$ and $\phi^{\prime}$ denote the angular coordinates of the point $\vec{r}^{\prime} . T_{p}$ is the Fresnel transmission function for $p$-polarized light,

$$
T_{p}=\frac{2 n_{1} \cos \theta^{\prime}}{n_{1} \cos \theta^{\prime}+n_{2} \cos \theta_{t}^{\prime}},
$$

and $T_{s}$ is the Fresnel transmission function for $s$-polarized light,

$$
T_{s}=\frac{2 n_{1} \cos \theta^{\prime}}{n_{2} \cos \theta^{\prime}+n_{1} \cos \theta_{t}^{\prime}} .
$$

The angle of transmission, $\theta_{t}^{\prime}$, is given through Snell's Law, or

$$
\theta_{t}^{\prime}=\sin ^{-1}\left(\frac{n_{2}}{n_{1}} \sin \theta^{\prime}\right)
$$

\subsection{Normally incident light}

The theoretical setup for this investigation is illustrated in Fig. 1. A plane wave is traveling within the microlens medium having a refractive index of $n_{2}$ and incident upon the spherical exit surface of the microlens. The curvature of the spherical exit surface is assumed to have a maximum half-angle of $\theta_{\max }$. Beyond the exit surface, the refractive index is assumed to be $n_{1}$, where $n_{2}>n_{1}$.

For incident plane waves traveling parallel to the $z$-axis, the electric and magnetic fields can be expressed as

$$
E(\vec{r}) \equiv A \vec{U}(\vec{r}),
$$

and

$$
H(\vec{r}) \equiv A \frac{n_{1}}{Z_{o}} \vec{V}(\vec{r}),
$$

where

$$
\vec{U}(\vec{r})=\int_{0}^{2 \pi} \int_{0}^{\theta_{m}} \sin \theta^{\prime} d \theta^{\prime} d \phi^{\prime}\left[\vec{u}_{1}+\vec{u}_{2}+\vec{u}_{3}\right]
$$

and

$$
\vec{V}(\vec{r})=\int_{0}^{2 \pi} \int_{0}^{\theta_{m}} \sin \theta^{\prime} d \theta^{\prime} d \phi^{\prime}\left[\vec{v}_{1}+\vec{v}_{2}+\vec{v}_{3}\right]
$$

with

$$
A=\frac{-i p_{1} T_{p}}{4 \pi} E_{o}
$$


where $p_{1}=2 \pi n_{1} R / \lambda$.

The angle $\theta_{m}$ is the maximum half-angle of the aperture, and $\vec{u}_{1}, \vec{u}_{2}, \vec{u}_{3}, \vec{v}_{1}, \vec{v}_{2}$ and $\vec{v}_{3}$ represent the three vectors of the three individual terms of $\vec{E}$ and $\vec{H}$ in Eqs. 1 and 2, respectively. Each of these vectors have the following components:

$$
\begin{aligned}
& u_{1 x}=f_{2} G_{1}\left(\cos \theta^{\prime} \cos ^{2} \phi^{\prime}+t_{1} \cos \theta_{t}^{\prime} \sin ^{2} \phi^{\prime}\right) \\
& u_{1 y}=f_{2} G_{1} \sin \phi^{\prime} \cos \phi^{\prime}\left(\cos \theta^{\prime}-t_{1} \cos \theta_{t}^{\prime}\right) \\
& u_{1 z}=-f_{2} G_{1} \sin ^{2} \theta^{\prime} \cos \phi^{\prime}, \\
& u_{2 x}=f_{2} G_{2}\left[\left(z_{1}-z_{1}^{\prime}\right) \alpha_{1 y}-\left(y_{1}-y_{1}^{\prime}\right) \alpha_{1 z}\right] \\
& u_{2 y}=f_{2} G_{2}\left[\left(x_{1}-x_{1}^{\prime}\right) \alpha_{1 z}-\left(z_{1}-z_{1}^{\prime}\right) \alpha_{1 x}\right] \\
& u_{2 z}=f_{2} G_{2}\left[\left(y_{1}-y_{1}^{\prime}\right) \alpha_{1 x}-\left(x_{1}-x_{1}^{\prime}\right) \alpha_{1 y}\right], \\
& u_{3 x}=f_{2} G_{2} \sin \theta_{t}^{\prime} \cos \phi^{\prime}\left(x_{1}-x_{1}^{\prime}\right) \\
& u_{3 y}=f_{2} G_{2} \sin \theta_{t}^{\prime} \cos \phi^{\prime}\left(y_{1}-y_{1}^{\prime}\right) \\
& u_{3 z}=f_{2} G_{2} \sin \theta_{t}^{\prime} \cos \phi^{\prime}\left(z_{1}-z_{1}^{\prime}\right), \\
& v_{1 x}= f_{2} G_{1} \sin \phi^{\prime} \cos \phi^{\prime}\left(t_{1} \cos \theta^{\prime}-\cos \theta_{t}^{\prime}\right) \\
& v_{1 y}=f_{2} G_{1}\left(t_{1} \cos \theta^{\prime} \sin \phi^{\prime}+\cos \theta_{t}^{\prime} \cos { }^{2} \phi^{\prime}\right) \\
& v_{1 z}=f_{2} G_{1}\left(t_{1} \sin \theta^{\prime} \sin \phi^{\prime}\right), \\
& v_{2 x}=f_{2} G_{2}\left[\left(z_{1}-z_{1}^{\prime}\right) \beta_{1 y}-\left(y_{1}-y_{1}^{\prime}\right) \beta_{1 z}\right] \\
& v_{2 y}=f_{2} G_{2}\left[\left(x_{1}-x_{1}^{\prime}\right) \beta_{1 z}-\left(z_{1}-z_{1}^{\prime}\right) \beta_{1 x}\right] \\
& v_{2 z}=f_{2} G_{2}\left[\left(y_{1}-y_{1}^{\prime}\right) \beta_{1 x}-\left(x_{1}-x_{1}^{\prime}\right) \beta_{1 y}\right],
\end{aligned}
$$

and

$$
\begin{aligned}
& v_{3 x}=f_{2} G_{2} t_{1} \sin \theta_{t}^{\prime} \sin \phi^{\prime}\left(x_{1}-x_{1}^{\prime}\right) \\
& v_{3 y}=f_{2} G_{2} t_{1} \sin \theta_{t}^{\prime} \sin \phi^{\prime}\left(y_{1}-y_{1}^{\prime}\right) \\
& v_{3 z}=f_{2} G_{2} t_{1} \sin \theta_{t}^{\prime} \sin \phi^{\prime}\left(z_{1}-z_{1}^{\prime}\right) .
\end{aligned}
$$

The function $f_{2}$ is the phase of the electromagnetic field at the point $\vec{r}^{\prime}$, or

$$
f_{2}=e^{-i p_{2} \cos \theta^{\prime}}
$$

and $G_{1}$ and $G_{2}$ are the functions:

$$
\begin{gathered}
G_{1} \equiv 4 \pi R G=\frac{e^{-i p_{2} \rho_{1}}}{\rho_{1}}, \text { and } \\
G_{2} \equiv-\frac{1}{i p_{2} \rho_{1}} \frac{\partial G_{1}}{\partial \rho_{1}}=\frac{G_{1}}{\rho_{1}}\left(1+\frac{1}{i p_{2} \rho_{1}}\right) .
\end{gathered}
$$

The parameter $t_{1}$ is the ratio of the Fresnel transmission function for $s$-polarized light to the transmission function for $p$-polarized light, or

$$
t_{1} \equiv \frac{T_{s}}{T_{p}}=\frac{n_{1} \cos \theta^{\prime}+n_{2} \cos \theta_{t}^{\prime}}{n_{1} \cos \theta_{t}^{\prime}+n_{2} \cos \theta^{\prime}} .
$$

The components of $\vec{\alpha}_{1}$ and $\vec{\beta}_{1}$ introduced in Eqs. 17 and 20 are given by:

$$
\begin{aligned}
& \alpha_{1 x}=\sin \phi^{\prime} \cos \phi^{\prime}\left(t_{1} \cos \theta^{\prime}-\cos \theta_{t}^{\prime}\right) \\
& \alpha_{1 y}=\cos \theta_{t}^{\prime} \cos ^{2} \phi^{\prime}+\cos \theta^{\prime} \sin ^{2} \phi^{\prime} \\
& \alpha_{1 z}=-t_{1} \sin \theta^{\prime} \sin \phi^{\prime},
\end{aligned}
$$


and

$$
\begin{aligned}
& \beta_{1 x}=-\cos \theta^{\prime} \cos ^{2} \phi^{\prime}-t_{1} \cos \theta_{t}^{\prime} \sin ^{2} \phi^{\prime} \\
& \beta_{1 y}=\sin \phi^{\prime} \cos \phi^{\prime}\left(t_{1} \cos \theta_{t}^{\prime}-\cos \theta^{\prime}\right) \\
& \beta_{1 z}=\sin \theta^{\prime} \cos \phi^{\prime} .
\end{aligned}
$$

The coordinates $x_{1}, y_{1}, z_{1}, x_{1}^{\prime}, y_{1}^{\prime}$, and $z_{1}^{\prime}$ can be expressed in terms of the spherical coordinates as

$$
\begin{aligned}
& x_{1}=r_{1} \sin \theta \cos \phi \\
& y_{1}=r_{1} \sin \theta \sin \phi \\
& z_{1}=r_{1} \cos \theta,
\end{aligned}
$$

and

$$
\begin{aligned}
& x_{1}^{\prime}=\sin \theta^{\prime} \cos \phi^{\prime} \\
& y_{1}^{\prime}=\sin \theta^{\prime} \sin \phi^{\prime} \\
& z_{1}^{\prime}=\cos \theta^{\prime},
\end{aligned}
$$

where $r_{1} \equiv r / R$.

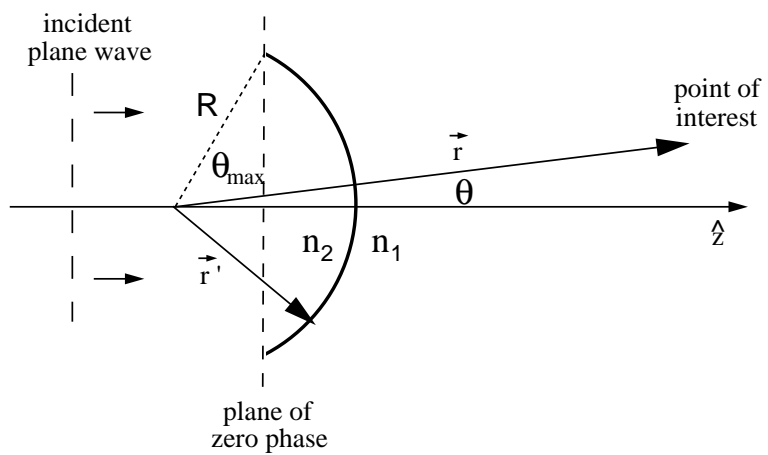

Figure 1. Theoretical setup for calculating light fields beyond the exit surface of a microlens. The plane wave is incident in the $+\hat{z}$ direction and refracted at the spherical exit surface with maximum half angle $\theta_{\text {max }}$, and radius of curvature $R$.

\subsection{Light incident at an angle}

Figure 2 illustrates the theoretical setup for light incident upon the spherical surface at an angle of $\gamma$ with respect to the $z$-axis. For ease of calculation, a second (double-primed) coordinate system is introduced such that $\hat{x}^{\prime \prime}$ and $\hat{z}^{\prime \prime}$ are rotated about $\hat{y}^{\prime}$ by the angle of incidence, $\gamma$, with respect to $\hat{x}^{\prime}$ and $\hat{z}^{\prime}$, and $\hat{y}^{\prime \prime}=\hat{y}^{\prime}$.

All of the equations described previously for normally incident plane waves are valid for light incident at an angle, except for the fact that they apply to the double-primed coordinate system; i.e., all of the "primes" in Eqs. 1-29 are replaced with "double-primes." The difficultly in using the double-primed coordinate system arises in the limits of the integrations. The limits of the integrations are determined by the shape of the exit surface in that coordinate system. In the primed coordinate system of Fig. 2 , the limits of integration are $\theta^{\prime}=0$ to $\theta_{m}$, and $\phi^{\prime}=0$ to $2 \pi$. In the double-primed coordinate system, the limits of integration are a complicated function of $\theta^{\prime \prime}$ and $\phi^{\prime \prime}$ due to the fact that $z^{\prime \prime}$ does not pass through the center of the exit surface and the exit surface is not angularly symmetric about $r^{\prime \prime}$ for all values of $\theta^{\prime \prime}$. Mathematically and computationally it would be desirable to perform the integrations over the primed coordinates. If an expression is found that transforms the double-primed coordinates to the primed coordinates, then the integration can be performed in the primed coordinate system where all of the variables and functions of $\vec{U}$ and $\vec{V}$ (Eqs. 12 and 13) are expressed in the primed coordinate system. 


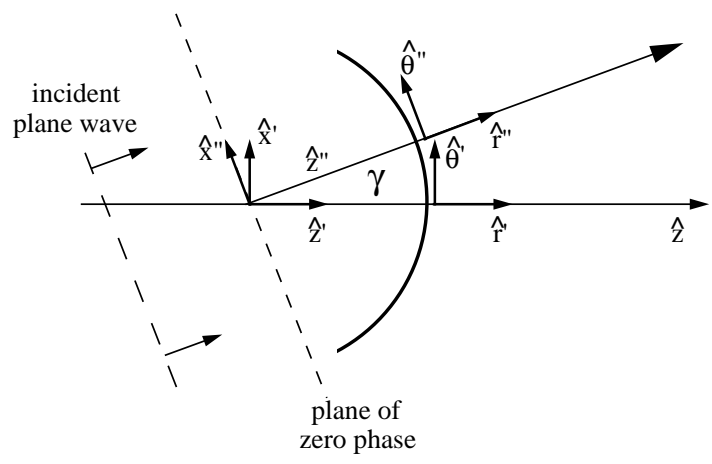

Figure 2. Theoretical setup for calculating light fields beyond the exit surface of a microlens. The plane wave is incident at an angle of $\gamma$ with respect to the $+\hat{z}$ direction.

The relationships between the primed and double-primed spherical coordinates can be found by first expressing the spherical primed coordinates in terms of the cartesian primed coordinates, or

$$
\begin{aligned}
& x^{\prime}=\sin \theta^{\prime} \cos \phi^{\prime} \\
& y^{\prime}=\sin \theta^{\prime} \sin \phi^{\prime} \\
& z^{\prime}=\cos \theta^{\prime} .
\end{aligned}
$$

Then the double-primed coordinate system is rotated about $\hat{y}^{\prime \prime}$ by an angle $-\gamma$ to become the primed cartesian coordinates:

$$
\begin{aligned}
& x^{\prime \prime}=x^{\prime} \cos \gamma-z^{\prime} \sin \gamma \\
& y^{\prime \prime}=y^{\prime} \\
& z^{\prime \prime}=x^{\prime} \sin \gamma+z^{\prime} \cos \gamma
\end{aligned}
$$

Substituting Eq. 29 into 30, we get

$$
\begin{aligned}
x^{\prime \prime} & =\left(\sin \theta^{\prime} \cos \phi^{\prime}\right) \cos \gamma-\left(\cos \theta^{\prime}\right) \sin \gamma \\
y^{\prime \prime} & =\sin \theta^{\prime} \sin \phi^{\prime} \\
z^{\prime \prime} & =\left(\sin \theta^{\prime} \cos \phi^{\prime}\right) \sin \gamma+\left(\cos \theta^{\prime}\right) \cos \gamma
\end{aligned}
$$

Expressing the double-primed spherical coordinates in terms of the double-primed cartesian coordinates results in

$$
\theta^{\prime \prime}=\cos ^{-1}\left(\cos \theta^{\prime} \cos \gamma+\sin \theta^{\prime} \cos \phi^{\prime} \sin \gamma\right)
$$

and

$$
\phi^{\prime \prime}=\tan ^{-1}\left(\frac{\sin \theta^{\prime} \sin \phi^{\prime}}{\sin \theta^{\prime} \cos \phi^{\prime} \cos \gamma-\cos \theta^{\prime} \sin \gamma}\right) .
$$

Equations 1-29 can now be used for light incident upon a microlens at an angle of $\gamma$ if the right hand side of Eqs. 32 and 33 are substituted for all of the " $\theta^{\prime \prime}$ " and " $\phi^{\prime \prime}$ " variables, respectively, in Eqs. 1-29.

\section{NORMAL INCIDENCE CALCULATIONS}

Figure 3 is a calculation of the modulus square of the electric field versus the on-axis distance from the center of curvature. The parameters chosen for this calculation are the same as those for the microlenses by Dumke et. $a l^{2}$ to optically trap cold ${ }^{85} \mathrm{Rb}$ atoms. The microlens array is an array of spherically curved surfaces on one side of a fused silica substrate $(n=1.454$ at $\lambda=780 \mathrm{~nm}$ ). The light enters the other (flat) side of the substrate and 
exits each spherical surface. The spherical surface has a radius of curvature of $R=312.5 \mu \mathrm{m}$ and a maximum half-angle of $\theta_{m}=11.5^{\circ}$. Using geometrical optics, the focal spot should be observed at an axial distance of $3.2 R$ from the center of curvature. The peak intensity calculated in Fig. 3 is found at an axial distance of 3.11 $R$. Figure 4 is a plot of the modulus square of the $x$-component of the electric field versus the radial position, at the focal plane $(z=3.11 R)$. One interesting feature of the on-axis intensity is the asymmetry before and after the focal region. The on-axis intensity modulations before the primary focal plane resemble the on-axis intensity characteristics for the diffraction of light by a circular aperture without a focusing medium. ${ }^{6,7}$ The on-axis intensity features before the focal spot indicate that light distributions in this region are influenced by the diffractive nature of the circular edge of the exit surface as well as the focal nature of the spherical shape of the exit surface. To further investigate the features before the focal plane, Figs. 5 and 6 are plots of the radial intensity at the locations of the on-axis minimum and maximum occurring just before the focal plane, respectively. The beam profiles calculated in Figs. 5 and 6 very closely resemble those of beam profiles calculated for diffraction of light by a simple circular aperture using scalar diffraction theory, ${ }^{6}$ and vector diffraction theory. 7,8

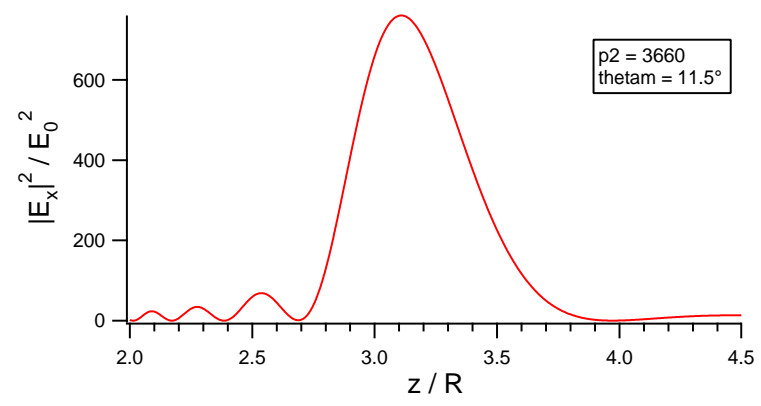

Figure 3. Modulus square of the $x$-component of the electric field versus axial position for a microlens with $2 \pi n R / \lambda=3660$ and a maximum half-angle of $11.5^{\circ}$.

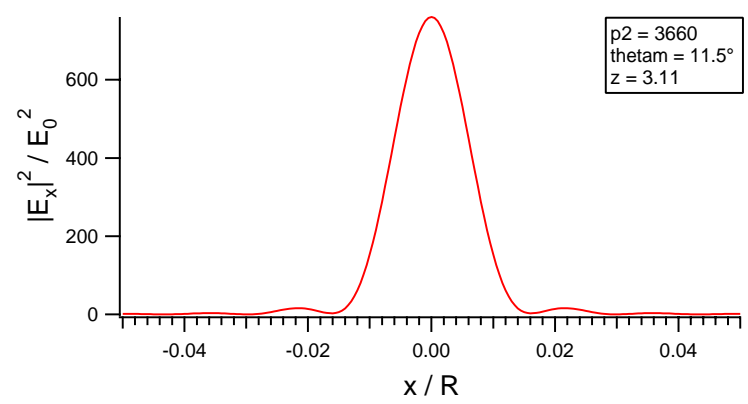

Figure 4. Modulus square of the $x$-component of the electric field versus radial position for a microlens with $2 \pi n R / \lambda=$ 3660 and a maximum half-angle of $11.5^{\circ}$.

\section{SPHERICAL ABERRATION}

Figure 7 is a plot of the on-axis intensity for a lens with a focal parameter of $p_{2}=2 \pi n_{2} R / \lambda=5 \times 10^{5}$ for a variety maximum half-angles. A lens parameter of $p_{2}=5 \times 10^{5}$ would correspond to a radius of curvature of $2.8 \mathrm{~cm}$ for $532-\mathrm{nm}$ light. For $p_{2}=5 \times 10^{5}$ the onset of the effects of spherical aberration are evident for even a maximum half-angle of $\theta_{m}=7.5^{\circ}$. As $\theta_{m}$ is increased from 5 to $7.5^{\circ}$ the maximum value of the on-axis intensity increases. Beyond a half-angle of $7.5^{\circ}$, the maximum on-axis intensity no longer increases in magnitude with increasing $\theta_{m}$. For $\theta_{m}=10^{\circ}$, significant deviations exist in the on-axis intensity profile from that of an aberration-free lens such 


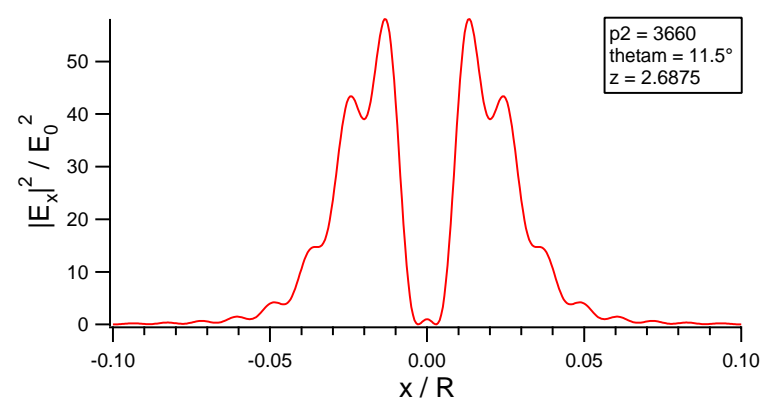

Figure 5. Modulus square of the $x$-component of the electric field versus radial position for a microlens with $2 \pi n R / \lambda=$ 3660 , a maximum half-angle of $11.5^{\circ}$, and an axial position of the on-axis minimum closest to the focal plane, or $z=2.69 R$.

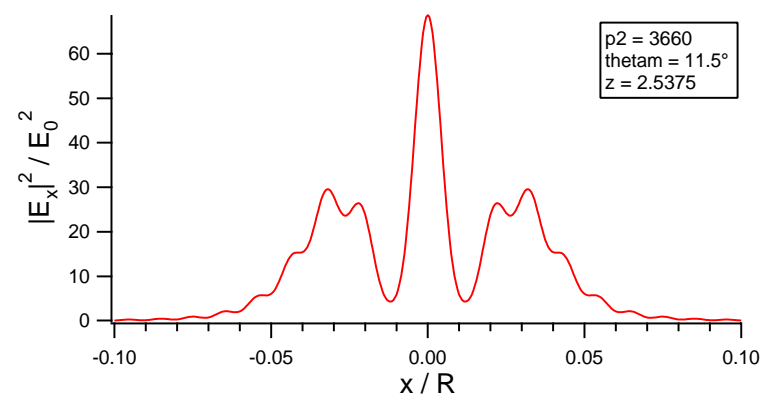

Figure 6. Modulus square of the $x$-component of the electric field versus radial position for a microlens with $2 \pi n R / \lambda=$ 3660 , a maximum half-angle of $11.5^{\circ}$, and an axial position of the first localized on-axis maximum closest to the focal plane, or $z=2.54 R$.

as that of the $\theta_{m}=5^{\circ}$ on-axis intensity plot. Note that this was not the case for a microlens with $p_{2}=3660$ and $\theta_{m}=11.5^{\circ}$, discussed in the previous section, illustrating that the onset of spherical aberration is dependent upon the ratio of the curvature of radius to the wavelength of light. The higher the ratio, the earlier the onset of spherical aberration for increasing maximum half-angles. In Fig. 7(b) the maximum half-angle is increased even further to investigate the effects of severe spherical aberration. Once the maximum on-axis intensity has saturated (around $7.5^{\circ}$ for $p_{2}=5 \times 10^{5}$ ), the region of higher intensities elongates along the axis of propagation towards the exit surface of the lens. The growth of the "focal spot" towards the exit surface of the lens can be explained using geometrical optics. According to geometrical optics, if a series of parallel rays are refracted by a spherical surface then the paraxial rays focus at the expected focal spot. Non-paraxial rays will be refracted by the spherical surface such that they will cross the optical axis at a point closer to the exit surface. The farther the initial ray is located from the optical axis, the closer to the surface of the lens the ray will cross the optical axis.

Figures $8(\mathrm{a})$ and (b) are plots of the radial intensity profile at the focal plane of a plane wave refracted by a spherical exit surface as a function of the maximum half-angle on a linear and a logarithmic scale, respectively. The intensity axis of Fig. 8 is normalized to the incident intensity before the spherical surface. Once the on-axis maximum intensity saturation point is reached for increasing $\theta_{m}$, no significant changes in the radial beam profile is observed on a linear scale for increasing $\theta_{m}$. Figure $8(\mathrm{~b})$ is the same plot as Fig. 8(a), except that the intensity scale is logarithmic. On a logarithmic scale it is apparent that the light collected by the outer rings of the spherical surface crosses the "focal" plane at off-axis locations; i.e., the energy collected by the outer regions of the spherical surface is propagated to off-axis locations in the focal plane. Away from the central focal region, the intensity of a spherically aberrated beam can be orders of magnitude higher than that of a non-aberrated beam. 

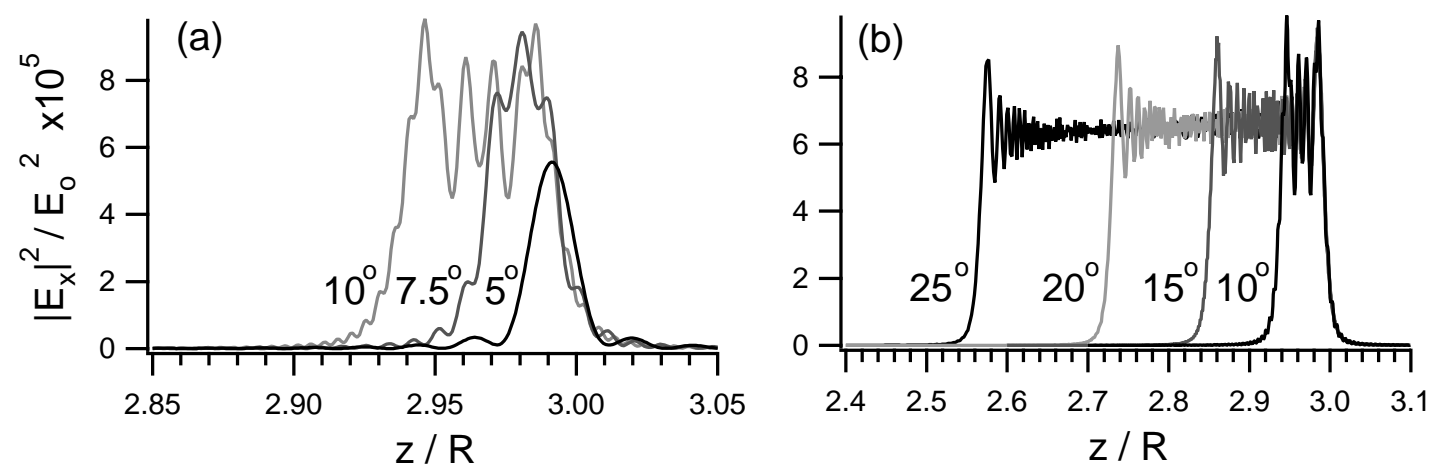

Figure 7. Calculated values of the intensity (normalized to the incident intensity) along the axis of the lens (normalized to the radius of curvature of the lens) for various values of the maximum half-angle $\theta_{m}\left(5^{\circ}, 7.5^{\circ}, 10^{\circ}, 15^{\circ}, 20^{\circ}\right.$, and $25^{\circ}$ ) of the lens surface for a normally incident plane wave with $2 \pi n R / \lambda=5 \times 10^{5}$.
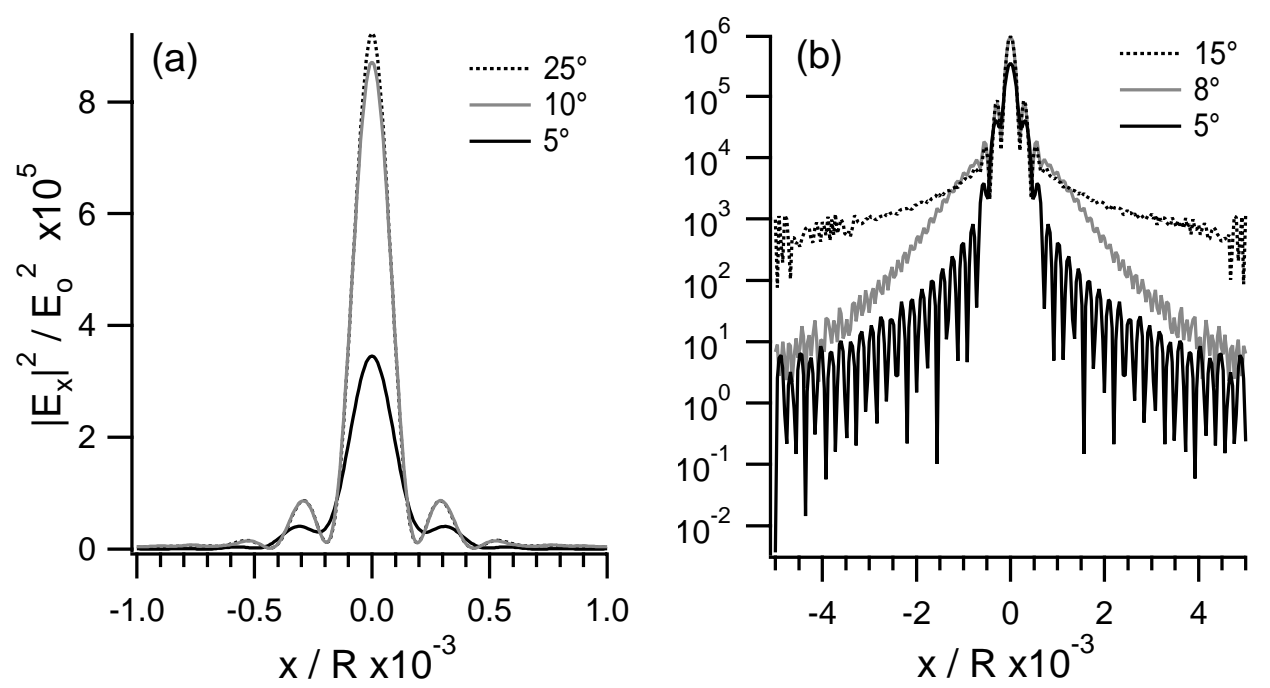

Figure 8. Calculated values of the focal plane intensity distributions (normalized to the incident intensity) along the $x$-axis (normalized to the radius of curvature of the lens) on a linear and long scale, respectively, for various values of the maximum half-angle $\theta_{m}\left(5^{\circ}, 8^{\circ}, 10^{\circ}, 15^{\circ}\right.$, and $\left.25^{\circ}\right)$ of the lens surface. The light is a normally incident plane wave with $2 \pi n R / \lambda=5 \times 10^{5}$, and the 'focal plane' occurs for $z / R=2.984$. 


\section{OFF-AXIS FOCUSING AND COMA}

Figure 9 is a calculation of the intensity of the light field versus $x$ and $y$ in the focal plane $(z / R=3.15$ for $2 \pi n_{2} R / \lambda=10^{4}$ ) and incident angles, $\gamma$, of 0,7 and $15^{\circ}$. With an incident angle of $7^{\circ}$ the effects of coma are observed as subtle partial rings on the far side of the focal spot, and a slight elongation of the focal spot in the direction of the incident angle. For an incident angle of $15^{\circ}$, severe coma is observed as a broadening of the focal spot in both the $x$ and $y$-directions in addition to conical beam fanning as expected according to geometrical optics. However, contrary to geometrical optics (which would predict a smooth intensity decay throughout the beam fanning) intensity calculations presented here predict the interference patterns arising from the wave nature of light. The degree of coma observed is also a function of the radius of curvature to wavelength ratio. Similar to Fig. 9, Fig. 10 is a series of intensity versus $x$ and $y$ images, where the only difference is that the value of $p_{2}$ has been decreased from $10^{4}$ to 3660 . For smaller $p_{2}$, the effects of coma for $\gamma=7^{\circ}$ have essentially been eliminated, and the coma effects for $\gamma=15^{\circ}$ have been significantly reduced, as compared to Fig. 9.

Figure 11 is a calculation of the beam intensity profile (along the $x$-axis) for the same parameters as that of Fig. 9, but a series of angles of incident leading up to $7^{\circ}$. The relative ranges for each graph are kept constant to aid in the visual comparison of the absolute intensities and radial beam fanning. Even for an angle of incidence of $3^{\circ}$ asymmetries in the beam profile are observed for $2 \pi n R / \lambda=10^{4}$. As the angle of incidence is increased, the overall maximum intensity decreases and the width of the focal spot increases as well as the amount of energy observed outside of the primary focal spot.
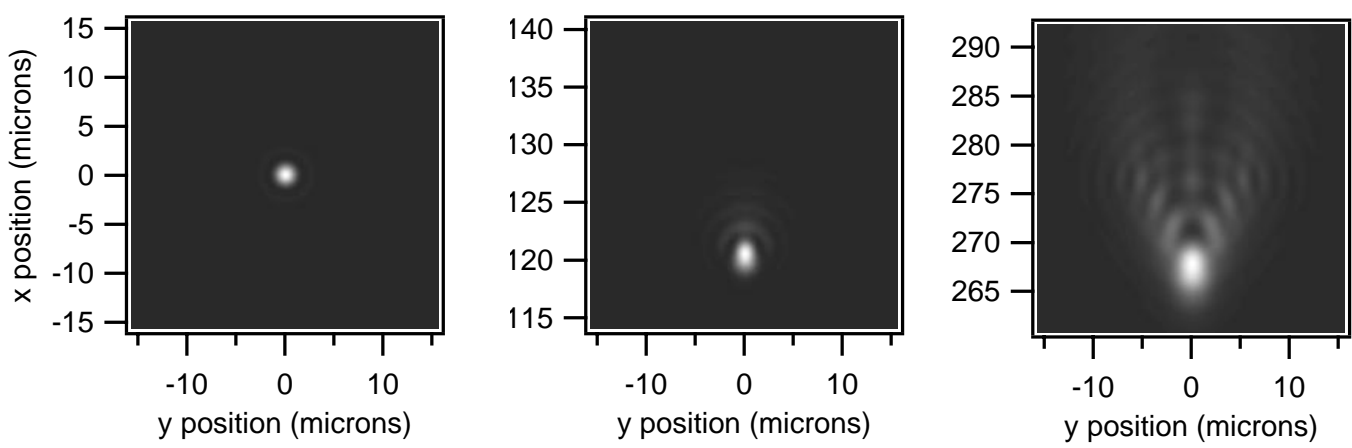

Figure 9. Calculated intensity distributions in the focal plane for $2 \pi n R / \lambda=10^{4}$ with $\theta_{m}=11.54^{\circ}, n=1.454$, and incident angles of $\gamma=0,7^{\circ}$ and $15^{\circ}$, respectively.
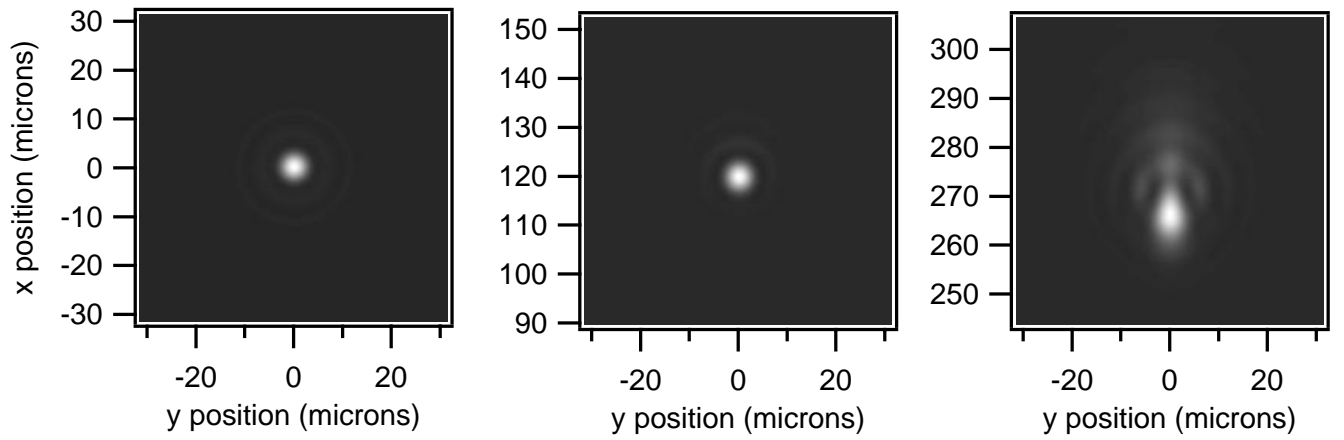

Figure 10. Calculated intensity distributions in the focal plane for $2 \pi n R / \lambda=3660$ with $\theta_{m}=11.54^{\circ}, n=1.454$, and incident angles of $\gamma=0,7^{\circ}$ and $15^{\circ}$, respectively. 

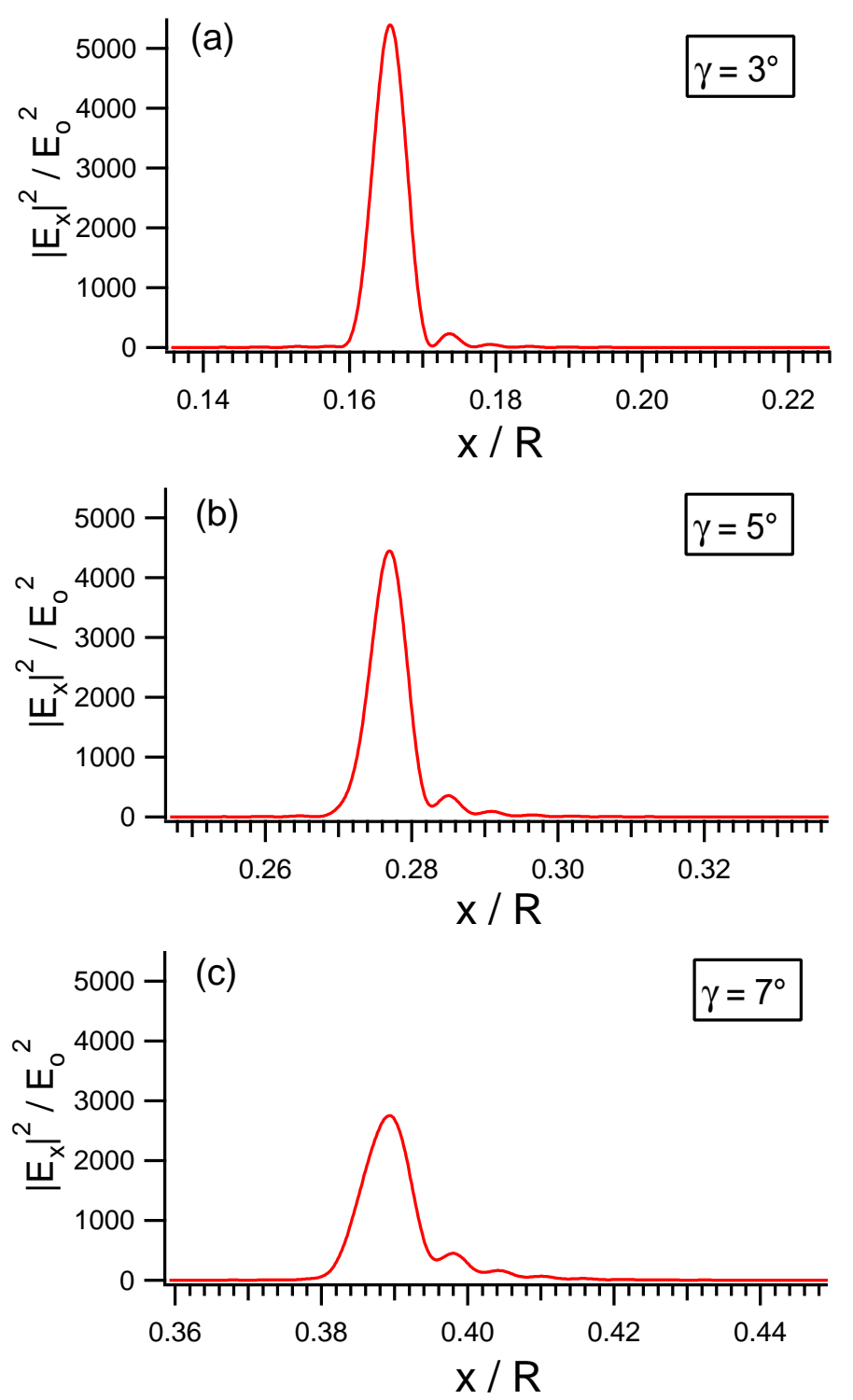

Figure 11. Effects of coma on the radial beam profile of a plane wave incident on a microlens spherical exit surface with $2 \pi n R / \lambda=10^{4}$ and $z / R=3.15$ at an angle of (a) $3^{\circ}$, (b) $5^{\circ}$, and (c) $7^{\circ}$. All horizontal and vertical scales are over the same ranges. 


\section{CONCLUSIONS}

A computational method has been employed to determine all of the vector electromagnetic field components for a region of space beyond a spherical microlens using vector diffraction theory. Using this method the field and intensity distributions are obtained and the onset and effects of spherical aberration and coma can be studied. The onset and severity of spherical aberration depend on both the maximum collection angle of the lens and the ratio of the radius of curvature to the wavelength of light. The onset and severity of coma depend on both the angle of incidence and the ratio of the radius of curvature of the lens to the wavelength of the incident light.

\section{REFERENCES}

1. J. E. Bjorkholm, R. R. Freeman, A. Ashkin, and D. B. Pearson, "Observation of focusing of neutral atoms by the dipole forces of resonance-radiation pressure," Phys. Rev. Lett. 41, 1361-1364 (1978).

2. R. Dumke, M. Volk, T. Müther, F. B. J. Buchkremer, G. Birkl, and W. Ertmer, "Micro-optical realization of arrays of selectively addressable dipole traps: a scalable configuration for quantum computation with atomic qubits," Phys. Rev. Lett. 89, 097903 (2002).

3. J. A. Stratton Electromagnetic Theory (McGraw-Hill Book Company, Inc., New York, NY 1941.)

4. J. A. Stratton and L. J. Chu, "Diffraction theory of electromagnetic waves," Phys. Rev. 56, 99 (1939).

5. E. J. Rothwell and E. J. Cloud, Electromagnetics (CRC Press, Baca Raton, FL 2001.)

6. G. D. Gillen and S. Guha, "Modeling and propagation of near-field diffraction patterns: a more complete approach," Am. J. Phys. 72, 1195 (2004).

7. S. Guha and G. D. Gillen, "Description of light propagation through a circular aperture using nonparaxial vector diffraction theory," Opt. Express 13, 1424 (2005).

8. G. D. Gillen, S. Guha, and K. Christandl, "Optical dipole traps for cold atoms using diffracted laser light," Phys. Rev. A 73, 013409 (2006). 